\title{
Hemangioma racemoso da retina: Um caso clínico
}

\section{Retinal Racemose Hemangioma: Case Report}

Filipe Mira Ferreira ${ }^{1}$, Augusto Ferreira Barbosa², Maria Catarina Santos Isabel Rodrigues Paiva ${ }^{3}$, António Jesus Roque Loureiro ${ }^{4}$

\section{$\underline{\text { RESUMO }}$}

O Hemangioma racemoso da retina é uma malformação congênita caracterizada por comunicações arteriovenovas (AV) retinianas. Em cerca de $30 \%$ dos casos pode haver manifestações a nível do sistema nervoso central (SNC), denominando-se então por Síndrome de Wyburn-Mason. Os autores apresentam um caso clínico de um doente do sexo masculino de 39 anos que recorre ao serviço de Urgência devido a diminuição da acuidade visual do olho esquerdo (OE) com cerca de seis dias de evolução. Apresenta melhor acuidade visual corrigida olho direito (OD): 4/10 e olho esquerdo (OE): 2/10. À fundoscopia apresenta disco óptico com limites indefinidos, alguma palidez, dilatação e tortuosidade, AV marcada bilateralmente. O hemangioma racemoso da retina é um diagnóstico clínico raro, efectuado por meio do exame oftalmológico. É importante verificar o envolvimento do SNC por meio da realização de angiorressonância magnética nuclear. Atualmente não existe nenhum tratamento preconizado, sendo importante a realização de observações periódicas para averiguação de eventuais complicações.

Descritores: Malformações arteriovenosas; Neoplasia da retina/diagnóstico; Hemangioma/diagnóstico; Síndrome; Relatos de casos

\section{Abstract}

Racemose Haemangioma ( $\mathrm{RH})$ is a congenital anomaly, characterized by retinal arteriovenous(AV) malformation. About 30\% of patients have signs of central nervous system (CNS) involvement, which has been called the Wyburn-Mason syndrome. The authors present a 39-year-old male patient, who was admitted at the emergency room with decreased visual acuity of his left eye, for the last 6 days. Ophthalmic examination was performed and showed a visual acuity of 4/10 in his right eye and of 2/10 in the left eye. Fundoscopy examination revealed pallor of both optic discs, with blurred limits and a characteristic dilation and AV connections. RH of retina is a rare entity which can be diagnosed by ophthalmic examination. Once this diagnosis is established, involvement of CNS has to be rulled out performing an angioMRI. Currently, no specific treatment has been defined, except a periodic follow-up to prevent complications.

Keywords: Arteriovenous malformation;Retinal neoplasms/diagnosis; Haemangioma/diagnosis; Syndrome; Case reports

\footnotetext{
${ }^{1}$ Interno do Internato Complementar de Oftalmologia do Centro Hospitalar de Coimbra - CHC- Coimbra - Portugal;

${ }^{2}$ Assistente Hospitalar Graduado de Oftalmologia do Centro Hospitalar de Coimbra - CHC - Coimbra - Portugal;

${ }^{3}$ Assistente Hospitalar de Oftalmologia do Centro Hospitalar de Coimbra CHC - Coimbra, Portugal;

${ }^{4}$ Diretor do Serviço de Oftalmologia do Centro Hospitalar de Coimbra - CHC Coimbra - Portugal.
}

Recebido para publicação em 5/11/2010 - Aceito para publicação em 28/3/2011 


\section{INTRODUÇÃO}

A s malformações arteriovenosas (AV) são alterações vasculares congênitas e raras, descritas pela primeira vez no século XIX ${ }^{(1)}$. Vários foram os termos usados para descrever estas malformações, desde angioma $\mathrm{AV}$, aneurisma $\mathrm{AV}$ e hemangioma racemoso $^{(2)}$. As malformações AV são um diagnóstico clínico caracterizado por comunicações AV, podendo ser classificadas segundo Archer et al. em 3 grupos ${ }^{(3)}$ :

Grupo I: comunicação AV com interposição de um plexo capilar anormal entre os vasos;

Grupo II: comunicações AV diretas ou múltiplas, sem leito capilar;

Grupo III: comunicações AV complexas e extensas, sem leito capilar.

Trata-se de um quadro clínico normalmente unilateral, idiopático, sem predileção por gênero ou raça, diagnosticado por meio de um exame de rotina, podendo haver sintomas visuais relacionados com a localização e extensão das lesões.

Em cerca de 30\% dos casos, existem também comunicações AV no sistema nervoso central, denominando-se Síndrome de Wyburn-Mason ${ }^{(4)}$.

Os autores apresentam um caso clínico de malformações AV bilaterais.

\section{Caso clínico}

Doente de 39 anos de idade, sexo masculino, raça caucasiana, recorre ao serviço de urgência devido a diminuição da acuidade visual em OE com cerca de seis dias de evolução.

Antecedentes pessoais de hipertensão arterial medicada e controlada com inibidor da enzima de conversão da angiotensina. Ao exame oftalmológico apresentava melhor acuidade visual corrigida para longe: OD: 4/10, OE: 2/10; Biomicroscopia: sem alterações relevantes; Tensão ocular (método aplanação): $12 \mathrm{mmHg}$ ODE; Fundoscopia: ODE: Disco óptico de limites indefinidos, edema ligeiro das fibras peripapilares, palidez ligeira (mais evidente no OE), dilatação e tortuosidade vascular associadas a malformações AV.

A angiografia fluoresceínica (AF) demonstrou hiperfluorescência papilar, grande tortuosidade vascular com compromisso arterial e venoso, bem como vasos dilatados em "saca-rolhas". Fase inicial apresentou enchimento simultâneo da rede arterial e venosa sem rede capilar interposta, sobretudo na arcada temporal superior e inferior. A fase venosa tardia demonstrou um padrão laminar venoso e um nervo óptico com trama vascular intensa (Figura 1-A). A ecografia ocular evidenciou elevação da cabeça do nervo óptico de aproximadamente $2 \mathrm{~mm}$ bilateralmente, ao passo que a tomografia de coerência óptica (OCT) evidenciou diminuição da camada de fibras nervosas peripapilares bilateralmente, embora mais marcado no OE. Os campos visuais 24-2 demonstraram uma ilha visão central e temporal superior no OD (Figura 1-B) e uma ilha visão temporal superior no OE.

Analiticamente não apresentava alterações no hemograma, PCR, bioquímica, microbiologia e imunoquímica. A realização de Tomografia computorizada (TC) orbitária e posteriormente angiorressonância magnética nuclear (RMN) orbitária e crânio-encefálica não demonstrou nenhuma alteração relevante. A realização de ecodoppler carotídeo bilateral também não demonstrou alterações. $\mathrm{O}$ doente foi observado pela Neurologia que descartou a presença de alterações neurológicas tendo posteriormente sido observado pela Hematologia que descartou a presença de distúrbios da coagulação.

Após follow up de seis meses, o doente mantém exame oftalmológico sobreponível continuando a ser observado periodicamente em consultas de retina médica.

\section{Discussão}

A etiologia das malformações AV permanece desconhecida, mas especula-se que estas apareçam devido a lesões ocorridas durante a embriogénese com consequente diferenciação anômala das células mesenquimatosas em células endoteliais ${ }^{(5)}$.

As malformações $\mathrm{AV}$ além de atingirem o olho, podem também atingir o SNC e mesmo outros orgãos( ${ }^{(6)}$, sendo mandatório o despiste de envolvimento do SNC através de avaliação pelo Neurologista e realização de angioRMN. Trata-se de um quadro clínico que se diagnostica durante a terceira década de vida, normalmente por meio de uma observação oftalmológica ocasional ou, mais raramente, por meio de diminuição da acuidade visual $^{(6)}$.O diagnóstico é efetuado por meio do exame oftalmológico, podendo ser auxiliado pela AF, OCT, entre outros. Na AF observamos em fase arterial precoce a comunicação directa entre artéria e veia, sem derrame tardio. Normalmente no OCT observa-se a diminuição da espessura da camada de fibras nervosas peripapilares.

No presente caso clínico existem malformações AV que se enquadram no grau II de Archer, com comunicação AV direta sem leito capilar interveniente. Trata-se de uma situação rara devido ao envolvimento ocular bilateral. A baixa da acuidade visual relatada pelo 


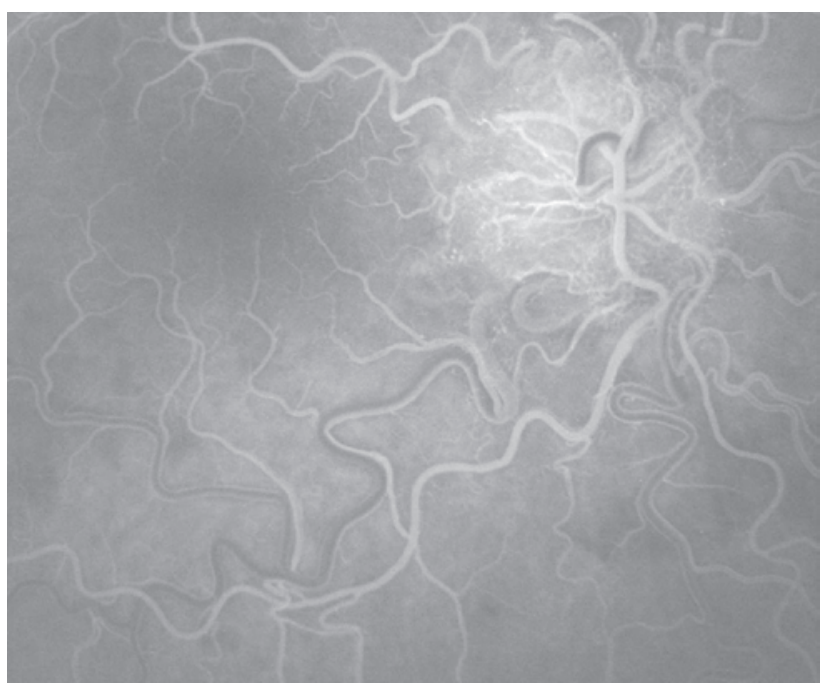

Figura1: A) AF em tempos precoces OD; B) Campos visuais 24-2 OD

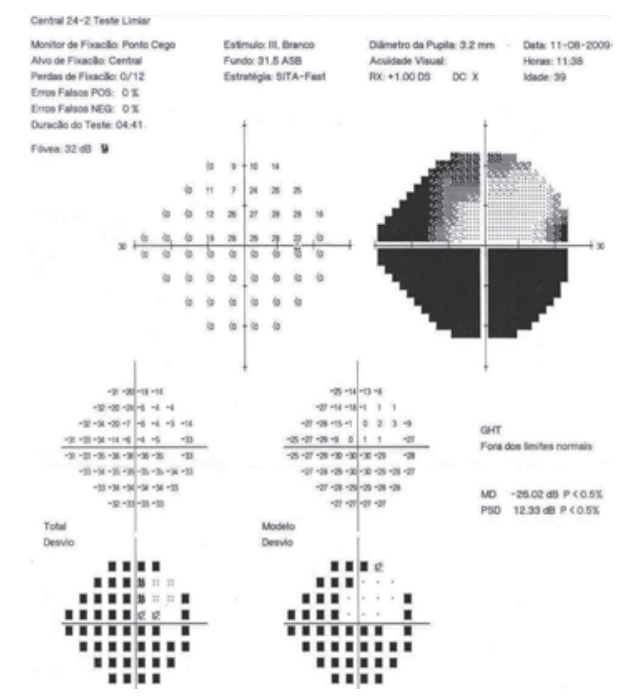

doente poderá ser explicada por meio de um corpo estranho que o doente refere ter sido removido uma semana antes à observação, na biomicroscopia atual não se encontraram alterações relevantes. Muitas vezes estes doentes conseguem efetuar as suas tarefas do quotidiano mesmo com baixas acuidades visuais, sendo o diagnóstico efetuado por meio de uma observação fundoscópica de rotina.O envolvimento do SNC foi descartado por meio da observação pela Neurologia e pela realização de AngioRMN. A observação oftalmológica, assim como a AF estão de acordo com o padrão típico da doença. Perante esta possibilidade diagnóstica é importante ter atenção com alguns diagnósticos diferenciais importantes, dos quais se destacam: telangiectasia peripapilar, hemangioma capilar, doença de Von Hippel Lindau e macrovasos retinianos congénitos ${ }^{(6)}$.

Normalmente, o alto fluxo e a elevada pressão hidrostática são responsáveis pela dilatação e tortuosidade venosas, situação que pode predispor a complicações oculares como sejam a hemorragia intrarretiniana, exsudação, formação de aneurisma, oclusão vascular, glaucoma neovascular e hemorragia do vítreo(7).

Os achados no exame oftalmológico, aliados aos resultados dos restantes exames auxiliares de diagnóstico, apontam como diagnóstico mais provável o hemangioma racemoso, a situação atípica observada neste caso clínico é o seu aparecimento bilateral, que é pouco frequente. Normalmente as lesões permanecem estáveis, não sendo necessário cumprir qualquer tratamento oftalmológico, preconizando-se uma observação oftalmológica periódica para despiste de eventuais complicações.
O prognóstico deste quadro clínico está dependente da localização e extensão das lesões, sendo sempre mandatório descartar o envolvimento do SNC por meio da solicitação de colaboração da Neurologia e realização de AngioRMN.

\section{REFERÊNCIAS}

1. Magnus H. Aneurysma arterioso-vensoum retinale. Virchows Arch Pathol Anat Physiol. 1874;60:38-45.

2. Pitault G, Haddad WM, Soubrane G. [Decompensation of a cirsoid aneurysm: a case report]. J Fr Ophtalmol. 2003;26(5):503-6. French.

3. Archer DB, Deutman A, Ernest JT, Krill AE. Arteriovenous communications of the retina. Am J Ophthalmol.1973;75(2):224-41.

4. Théron J, Newton TH, Hoyt WF. Unilateral retinocephalic vascular malformations. Neuroradiology. 1974;7(4):185-96.

5. Brown GC, Donoso LA, Magargal LE, Goldberg RE, Sarin LK. Congenital retinal macrovessels. Arch Ophthalmol. 1982;100(9):1430-6.

6. Mansour AM, Walsh JB, Henkind P. Arteriovenous anastamoses of the retina. Ophthalmology. 1987;94(1):35-40.

7. Gass JDM. Stereoscopic atlas of macular diseases: diagnosis and treatment. 4th ed. St. Louis: Mosby; 1997. Vol. 1. p. 440-2.

\author{
Autor correspondente \\ Filipe Mira Ferreira \\ Centro Hospitalar de Coimbra - Quinta dos Vales, $\mathbf{n}^{\mathbf{0}}$ \\ 3041 - 801 S. Martinho do Bispo, \\ Coimbra, Portugal \\ filipemiraferreira@gmail.com
}

